FORMATION Formation emploi

Revue française de sciences sociales

99 | juillet-septembre 2007

Les usages sociaux de la compétence

\title{
Une redéfinition des politiques de formation. Le cas de l'apprentissage dans les grandes entreprises
}

Redefining vocational training policies: the case of vocational training at large firms

Neuregelung der Berufsbildungspolitik. Lehrlingsausbildung in Großbetrieben

\section{Prisca Kergoat}

\section{(2) OpenEdition}

Journals

Édition électronique

URL : http://journals.openedition.org/formationemploi/1428

DOI : 10.4000/formationemploi.1428

ISSN : 2107-0946

Éditeur

La Documentation française

Édition imprimée

Date de publication : 1 juillet 2007

Pagination : 13-26

ISSN : 0759-6340

\section{Référence électronique}

Prisca Kergoat, « Une redéfinition des politiques de formation. Le cas de l'apprentissage dans les grandes entreprises », Formation emploi [En ligne], 99 | juillet-septembre 2007, mis en ligne le 30 septembre 2009, consulté le 10 décembre 2020. URL : http://journals.openedition.org/ formationemploi/1428; DOI : https://doi.org/10.4000/formationemploi.1428 


\section{DOSSIER}

\section{Une redéfinition des politiques de formation. Le cas de l'apprentissage dans les grandes entreprises}

Par Prisca Kergoat*

\section{Les politiques de formation par apprentissage menées par de grandes entreprises sont révélatrices des changements à l'œuvre depuis plus de deux décennies. L'apprentissage devient un lieu d'expérimentation posant ainsi les fondements d'une nouvelle relation en matière de formation, d'emploi et de qualification.}

Des changements en matière de politique de formation sont à l'œuvre depuis plus de deux décennies (Ropé, Tanguy, 1994). Nous nous proposons ici d'examiner quelques dimensions de ces changements. Nous montrons que les qualités sociales (nommées selon les cas savoir-être ou compétences comportementales), prépondérantes dans le cadre des formations par apprentissage, deviennent une composante de la connaissance à côté des savoirs et des savoir-faire. Cette rupture avec les normes établies de longue date est permise non seulement par la codification de la formation et du travail en termes de compétences mais encore par la mise en œuvre de politiques de contractualisation. Nous démontrons que ceci contribue à faire de l'apprentissage un lieu d'expérimentation en posant les fondements d'une nouvelle relation en matière de formation, d'emploi et de qualification.
Le sens et les effets de la substitution de la notion de formation à celle d'éducation et ce que ce changement contenait n'ont été que peu soulignés : la mise

* Prisca Kergoat est sociologue, maîire de conférences au centre de formation J.-F. Champollion (Albi), membre du CERTOP (Centre d'étude et de recherche travail-organisation-pouvoir) université de Toulouse Le Mirail. Ses domaines d'investigation sont le travail et son organisation, la formation professionnelle, la gestion du personnel, les politiques publiques, la socialisation des jeunesses populaires au travail. Elle a notamment publié : Kergoat P. (2006), « De l'indocilité au travail d'une fraction des jeunesses populaires. Les apprentis et la culture ouvrière ", Sociologie du travail, $n^{\circ}$ 48, pp. 545-560. KergoatP. (2002), "Le recrutement des apprentis en entreprise publique», in Les patrons, l'État et la formation des jeunes, Moreau G. (coord.), Paris, La Dispute, pp. 153-164. 
en correspondance de la formation avec l'emploi. La locution « relation formation/emploi», ainsi traduite dans un contexte historique à des fins particulières, a progressivement été naturalisée et oriente aujourd'hui les politiques éducatives (Tanguy, 2002). En même temps que la notion de formation primait sur celle d'éducation, elle se définissait en termes de compétences : une préférence récurrente est dès lors accordée à la notion de « compétence » au détriment de «savoirs disciplinaires» dans le domaine de l'éducation ${ }^{1}$ et de "qualification » dans le monde du travail² (Kergoat, 2001).

La rénovation de l'apprentissage et son extension aux professions intermédiaires se sont opérées dans ce cadre ( $c f$. encadré $\mathbf{1})$. L'examen de différentes formes d'apprentissage préparant aussi bien à des diplômes professionnels (CAP - certificat d'aptitude professionnelle ou BEP - brevet d'études professionnelles, baccalauréat professionnel), que techniques (BTS - brevet de technicien supérieur; DUT diplôme universitaire de technologie) montre que celles-ci constituent autant d'expériences visant à définir et à évaluer des contenus de formation en rupture avec ceux développés antérieurement. L'observation des politiques d'apprentissage mises en œuvre par les directions nationales de grandes entreprises permet également d'éclairer un autre aspect des changements politiques en cours, leur caractère contractualisé. Ce mouvement ayant été abordé dans les études sur la régionalisation (Lamanthe et Verdier, 1999) ou encore par une réflexion socio-historique (Tanguy, 2005), nous voudrions ici contribuer à une investigation générale et systématique du passage d'une politique nationale et étatique de l'apprentissage à une politique de concertation et de contractualisation. À cet effet, nous nous appuierons sur des cas concrets tels que les dispositifs d'apprentissage de trois grandes

\footnotetext{
${ }^{1}$ C'est dans les années 80 que la notion de compétence (se décomposant en savoir et en savoir-faire) apparaît dans les textes officiels de l'Éducation nationale. Parmi les actions impulsées par ce ministère pour infléchir les contenus enseignés, on soulignera la mise en place d'une Charte des programmes. Celle-ci énonce les principes qui organisent et légitiment le passage à un enseignement défini par, et visant à, la production des compétences vérifiables dans des situations et des tâches spécifiques. Cette charte procède à une définition des programmes, en termes de référentiels pour l'enseignement technique et professionnel, à partir des compétences qui leur sont associées. Ce référentiel est donc présenté comme un outil permettant de mettre en correspondance étroite offre de formation et distribution des activités professionnelles.

2 Dans le monde de l'entreprise, l'appel à la notion de compétence (se décomposant en savoir, savoir-faire et savoir-être) s'effectue corrélativement à la mise en place de politiques orientées vers la recherche de flexibilité, tant dans le domaine de l'emploi que dans celui de l'organisation du travail et de la gestion du personnel. Les compétences requises pour tenir un emploi sont identifiées au moyen de référentiels construits dans la même logique que ceux utilisés dans l'enseignement technique et professionnel. Introduit par l'Accord Cap 2000 (Usinor-Sacilor) en 1990, l'usage des compétences permet aux entreprises de fixer de nouvelles modalités de recrutement, de promotion et de rémunération. Soit autant d'applications qui président à la détermination des relations salariales jusqu'ici orchestrées par la notion de qualification, ellemême codifiée dans le cadre de négociations collectives.
}

\section{Encadré 1 \\ Rénovation de l'apprentissage}

Traditionnellement, les formations par apprentissage sont cantonnées aux petites entreprises. Depuis le XIXe siècle, en matière de formation initiale, les grandes entreprises industrielles se sont tournées vers l'enseignement technique et professionnel scolarisé (les écoles nationales professionnelles, les écoles pratiques de commerce et d'industrie ainsi que les écoles professionnelles publiques) ou ont développé, en leur sein, des écoles d'entreprise (ou "écoles de métiers »). C'est le cas de l'UIMM (Union des industries métallurgiques et minières) avec les écoles Schneider, Peugeot, Citroën, Berliet, Renault ainsi qu'EDF-GDF (Électricité de France-Gaz de France) qui recrutaient sur concours des élèves appelés à occuper des postes d'ouvriers qualifiés, voire de l'encadrement intermédiaire. Ces écoles partageaient avec la direction de l'enseignement technique l'idée qu'une «formation méthodique et complète » était nécessaire mais s'en séparaient sur un point : le lieu d'acquisition des savoirs ne pouvait être, selon elles, que l'entreprise (Hatzfeld, 1989 ; Brucy, 1998).

À partir des années 60 , si les valeurs fondatrices perdurent, ces écoles sont confrontées à des changements, dont les évolutions des politiques économiques et sociales de l'entreprise et le développement des formations techniques d'État (Quenson, 2001 ; Kergoat, 2002), qui vont conduire à leur fermeture. 


\section{Encadré 1 (suite)}

À la fin des années 80, l'apprentissage est concurrencé par l'enseignement professionnel scolarisé (lycée professionnel) et les formations par alternance telles que les contrats de qualification et les stages d'initiation à la vie professionnelle. La crise de l'apprentissage, le chômage, la recherche par l'Éducation nationale de moyens pour revaloriser la formation professionnelle mais également l'appel du CNPF (Centre national du patronat français) à un partenariat avec l'École, la réhabilitation de l'entreprise et l'idée progressivement admise que les compétences ne peuvent être produites et évaluées qu'en situation de travail amènent à développer la concertation sociale autour de l'apprentissage.

Cette troisième réorganisation de l'apprentissage (qui fait suite à celle de 1971) est d'abord une initiative patronale de l'UIMM notamment. De fait, la loi de 1987 est l'aboutissement d'un compromis entre différents acteurs: l'Éducation nationale, qui poursuit un objectif de valorisation de l'apprentissage et d'élévation du niveau de formation, le ministère du Travail qui souhaite que l'apprentissage ne devienne pas un concurrent des contrats de qualification, et le patronat qui recherche une maind'œuvre ayant une formation plus adaptée aux nouvelles formes d'organisation du travail et de gestion du personnel. L'apprentissage fait dès lors l'objet de politiques étatiques qui visent à le rénover et à le promouvoir, à parité avec les formations scolaires, tout en favorisant son introduction au sein des grandes entreprises; à cet effet, les entreprises bénéficient de primes et ont la possibilité de créer des CFA (Centre de formation d'apprentis) qui, à leur tour, peuvent passer des conventions avec des établissements d'enseignement privés et publics. Ces politiques étatiques permettent aux entreprises privées et publiques de former à l'ensemble des diplômes relevant du secteur industriel comme du secteur tertiaire, du CAP (certificat d'aptitude professionnelle) au diplôme d'ingénieur. De même, suite à la loi quinquennale de 1993, les régions peuvent définir une politique de formation professionnelle adaptée aux besoins locaux. Ces diverses transformations conduisent tout d'abord à une progression importante des effectifs d'apprentis (+ 260000 nouveaux contrats d'apprentissage ont été enregistrés en 2005 contre 130000 en $1992\left({ }^{*}\right)$ ) ainsi qu'à un renforcement de l'investissement des moyennes et des grandes entreprises dont celles du secteur public.

\section{Les employeurs utilisateurs de l'apprentissage $\left(^{* *}\right)$ en $\%$}

\begin{tabular}{|c|c|c|c|}
\hline & 1992 & 2000 & 2005 \\
\hline 0 à 49 salariés & 93 & 87 & 82 \\
\hline 50 à 249 salariés & 4 & 7 & 8 \\
\hline 250 salariés et plus & 3 & 6 & 10 \\
\hline
\end{tabular}

Enfin, l'objectif, présenté en 2004 dans le Plan de cohésion sociale, est de faire passer de 350000 à 500000 le nombre d'apprentis d'ici à 2009 et de parvenir à un seuil de $2 \%$ d'apprentis dans les entreprises de plus de 100 salariés, au lieu de 0,5\% actuellement. Pour cela, le gouvernement a mis en place différentes mesures destinées à rendre plus attractif l'apprentissage pour les jeunes comme pour les entreprises.

(*) : DARES, « L'apprentissage en 2005 : une nouvelle hausse des entrées », Premières Synthèses, mars 2007. $(* *)$ : DARES, « Les entrées en contrat d'apprentissage depuis $1992 », 2005$.

entreprises ( $c f$. encadré 2), en privilégiant une analyse des pratiques.

Nous interrogerons d'abord deux dimensions des dispositifs d'apprentissage étudiés : le recrutement ainsi que les contenus de formation et leurs évalua- tions. Nous chercherons à démontrer que les qualités sociales contribuent :

- à créer un nouveau sas de sélection ainsi qu'un nouveau mode de préparation au travail ;

- à dévaluer les savoirs et les savoir-faire mobilisés par les apprentis ; 


\section{Encadré 2 \\ Terrain et méthodes}

Les propositions formulées dans cet article sont issues de nos travaux let initialement d'une thèse, Kergoat, 2002) sur l'apprentissage dans les grandes entreprises. Nous nous attacherons ici à décrire la mise en place de l'apprentissage dans trois de ces dernières : deux grandes entreprises publiques et une entreprise privée et pour cinq formations (du CAP au BTS) (*) :

\begin{tabular}{|c|c|c|c|}
\hline Entreprises & Formations & Activités & $\%$ de femmes \\
\hline $\begin{array}{c}\text { Entreprise A } \\
\text { Publique, tertiaire }\end{array}$ & $\begin{array}{l}\text { CAP Tri-Acheminement-Distribution (TAD) } \\
\text { BTS Force de vente/ } \\
\text { DUT Technique de commercialisation (**) }\end{array}$ & $\begin{array}{c}\text { Facteurs/trieurs } \\
\text { Conseillers financiers }\end{array}$ & $\begin{array}{l}57 \\
70\end{array}$ \\
\hline $\begin{array}{c}\text { Entreprise B } \\
\text { Publique, industrielle }\end{array}$ & $\begin{array}{c}\text { BEP Électrotechnique }\left({ }^{* * *}\right) \\
\text { Bac pro Services }\end{array}$ & $\begin{array}{c}\text { Électriciens } \\
\text { Conseillers clientèle }\end{array}$ & $\begin{array}{c}1 \\
70\end{array}$ \\
\hline $\begin{array}{c}\text { Entreprise C } \\
\text { Privée, industrielle }\end{array}$ & $\begin{array}{l}\text { BTS Mécanique } \\
\text { et Automatismes Industriels (MAl) }\end{array}$ & $\begin{array}{c}\text { Techniciens } \\
\text { de maintenance }\end{array}$ & 0,5 \\
\hline
\end{tabular}

Ces trois très grandes entreprises ont des points communs. Elles ont initié, au début des années 90 et pour la première fois de leur histoire, une politique d'apprentissage orchestrée par les directions nationales puis mise en œeuvre par les services des ressources humaines et leur(s) CFA (Centre de formation d'apprentis). Parallèlement, elles restructurent leur organisation du travail et leur gestion du personnel autour de la notion de compétence et non plus de celle de qualification.

Une centaine d'entretiens ont été réalisés auprès des différents acteurs du dispositif (responsables formation ou RH (ressources humaines), équipes de travail, tuteurs, recruteurs, enseignants, etc.) dont 45 entretiens approfondis et répétés auprès d'apprentile)s.

Ces différents entretiens ont été rapprochés de documents d'entreprise permettant une mise en œuvre technique de l'action: guides de recrutement, de tutorat, référentiels de formation, d'emploi, de compétence (et de diplôme) et grilles d'évaluation des compétences. Nous avons également effectué de nombreuses observations in situ de différentes situations d'apprentissage (recrutement, travail, formation en entreprise ou en milieu scolaire ou encore évaluation du déroulement de la formation et de la concertation entre l'ensemble des acteurs).

Concernant les politiques de contractualisation, les différents entretiens ont été confrontés avec des documents permettant l'élaboration de l'action (lois, décrets, circulaires, etc.), sa mise en œuvre (comptes-rendus de réunion en entreprise ou en CFA, conventions de partenariat, etc.) ou encore sa diffusion (dossier présentant les structures, fiches de préconisation, etc.).

Ces données ont été resituées dans une dimension socio-historique grâce au dépouillement des archives des anciennes écoles de métiers de l'entreprise B. Enfin, nous nous sommes appuyé sur des données statistiques produites par ces trois entreprises, ainsi que sur des études du Céreq.

$\left(^{*}\right)$ : Respectivement certificat d'aptitude professionnelle et brevet de technicien supérieur.

$(* *)$ : diplôme universitaire de technologies.

$(* * *)$ : brevet d'études professionnelles.

- à réhabiliter la notion de discipline, disparue de l'École et de l'entreprise depuis près de deux décennies, en lui conférant un nouvel habillage.

Ensuite, nous examinerons les concertations mises en œuvre d'une part entre les grandes entreprises et les régions et d'autre part avec le ministère de l'Éducation nationale. Nous montrerons :
- que les évolutions décrites sont rendues possibles par les politiques de contractualisation qui permettent de contourner et/ou d'infléchir les normes établies de longue date ;

- que ces grandes entreprises tendent à remettre en question le rôle déterminant conféré aux diplômes par l'Éducation nationale dans l'organisation de 
la formation professionnelle et plus largement dans la production des hiérarchies sociales et professionnelles.

\section{CHANGER LES SALARIÉS PLUTÔT QUE LE TRAVAIL3}

Nous nous interrogerons donc d'abord sur le recrutement des apprentis puis sur les contenus de formation. Nous chercherons à comprendre les raisons pour lesquelles les entreprises privilégient les qualités sociales au détriment des savoirs et des savoir-faire détenus par les candidats à l'apprentissage et mis en jeu au sein des métiers préparés. En effet, ceci paraît d'autant plus étonnant que toute une littérature souligne l'importance des savoir-faire transmis en situation de travail (Stroobants, 1993).

\section{Un sas de sélection axé sur les itinéraires biographiques}

L'importance accordée au recrutement dans les deux entreprises publiques ( $\mathrm{A}$ et $\mathrm{B}$ ) est due au fait qu'il s'agit non seulement de sélectionner un apprenti mais aussi un futur salarié. C'est ainsi que dans l'entreprise B les trois différentes étapes du recrutement se conjuguent à une forte sélection qui avoisine, du moins à un niveau quantitatif, celle des Grandes Écoles ( $c f$. tableau 1) :

Tableau 1

Sélection des candidats à l'apprentissage

\begin{tabular}{|c|c|c|c|c|}
\hline & $\begin{array}{c}\text { Nombre } \\
\text { de dossiers } \\
\text { présentés }\end{array}$ & $\begin{array}{c}\text { Nombre } \\
\text { de candidats retenus } \\
\text { pour les tests }\end{array}$ & $\begin{array}{c}\text { Nombre } \\
\text { de candidats retenus } \\
\text { pour les entretiens }\end{array}$ & $\begin{array}{c}\text { Nombre } \\
\text { de candidats } \\
\text { sélectionnés }\end{array}$ \\
\hline $\begin{array}{c}\text { Total sur l'ensemble } \\
\text { des formations }\end{array}$ & 655 & $\begin{array}{c}223 \\
\text { soit } 34 \%\end{array}$ & 117 & 26 \\
soit $18 \%$ & $4 \%$ \\
\hline
\end{tabular}

Source : données recomposées par nos soins à partir des statistiques délivrées par l'entreprise B pour une des quatre zones de la région parisienne, 1999.

Ces deux entreprises, plus précisément les directions des ressources humaines, ont la totale maîtrise du dispositif de recrutement, puisque les établissements de l'Éducation nationale chargés d'une partie de la formation des apprentis n'interviennent pas. En ce sens, elles renouent avec les pratiques des écoles d'entreprise. Toutefois, si la sélection est tout aussi importante, elle ne s'opère plus sur concours évaluant essentiellement des savoirs formalisés mais s'organise autour de l'entretien de recrutement destiné à observer les qualités sociales des candidats.

- Une première sélection sur dossiers (comprenant dans certains cas une lettre de motivation) permet de vérifier si les candidats remplissent les conditions administratives (niveau de diplôme, âge). Selon le nombre de dossiers sélectionnés, d'autres critères peuvent intervenir tels que la résidence géographique, l'origine ethnique, ou encore le sexe des candidats.

\footnotetext{
${ }^{3}$ Ce titre fait référence à un article de Danièle Linhart, 1994.
}

- Une deuxième sélection (pour les CAP, BEP et les bac pro) s'opère à partir de tests évaluant les capacités logiques du candidat, ses connaissances en mathématiques et en français. Pour reprendre les propos de ces entreprises, l'objectif est de s'assurer que les candidats ont bien acquis les savoirs minimaux (lire, compter, raisonner).

- La troisième et dernière étape de la sélection (sur laquelle nous allons nous arrêter plus longuement) consiste en un entretien du candidat auprès d'un jury d'entreprise (organisé pour la circonstance) comprenant jusqu'à quatre personnes aux statuts divers (spécialistes du recrutement, cadres de la filière d'activité concernée ou des ressources humaines, voire formateurs ou tuteurs chargés de l'encadrement des apprentis).

Lors de cette troisième étape, des outils sont mis à disposition des membres du jury afin de départager et de hiérarchiser les candidats à partir des «qualités » détenues par ces derniers (Dubernet, 1996) : les outils de sélection se fondent sur les 
notions de «personnalité », de «motivation» et d'《expérience » ${ }^{4}$.

Pour les candidats se présentant à des formations préparant à des emplois d'exécution, les recruteurs pointent les expériences de précarité ; elles concernent aussi bien les périodes de chômage que l'occupation d'emplois précaires (vendeur ou livreur dans la restauration rapide, caissière ou technicien de surface, etc.). L'examen des activités professionnelles se limite, bien souvent, aux capacités du candidat à se mobiliser face à des situations instables (succession d'emplois différents), à se confronter à des conditions de travail difficiles (pénibilité physique, flexibilité des horaires de travail). C'est à partir de ce premier examen que les recruteurs apprécient le rapport des candidats à un système de moralité (ponctualité, probité, rapport au travail) et à un ordre social établi (capacité à tenir sa place vis-à-vis de la hiérarchie ou dans une équipe de travail).

Pour les candidats se présentant à des formations préparant à des emplois de maîtrise, ce sont les épreuves, les challenges qui sont appréhendés afin d'évaluer leur résistance au stress et à la compétition. La passation de concours, la pratique d'un sport individuel (tel que le tennis pour les jeunes hommes, la danse pour les jeunes filles) sont fortement valorisées. Il s'agit aussi d'observer le comportement du candidat dans son rapport au corps et/ou à autrui : maintien, tenue, élocution, affabilité, capacité à convaincre, à ne pas se laisser déstabiliser, à sourire au bon moment, etc.

Toutes ces notions (l'expérience, la motivation, la personnalité) ont pour caractéristiques communes d'une part, d'attribuer à des dimensions personnelles ce qui est avant tout le fruit d'un itinéraire individuel marqué par les appartenances sociales (de classe, de sexe et d'ethnie) ; d'autre part, de renvoyer à ce que

\footnotetext{
${ }^{4}$ La «personnalité » est définie comme « des qualités [...], des caractéristiques personnelles qui puissent être favorables à l'intégration $d u$ candidat à la fonction recherchée»; la " motivation » permet «d'identifier les forces qui animent ou les résistances qui risquent de freiner l'adaptation du candidat envers l'entreprise et le métier 》; 1' " expérience » doit permettre « d'explorer les différentes situations vécues par le candidat $q u$ 'elles soient scolaires, professionnelles, extra-professionnelles ou le cas échéant militaires, pour repérer les modes habituels de comportement et les attitudes, son ouverture sociale et intellectuelle » (Guide du recrutement des apprentis, entreprise A).
}

Pierre Bourdieu (1979) nomme des «habitus»: un système de dispositions acquises, permanentes et génératrices de conduites ${ }^{5}$.

L'importance accordée à des dispositions acquises individuellement par des acteurs tout au long de leur itinéraire :

- Prend appui sur une conception fortement naturalisée : à l'inverse des savoirs et des savoirfaire, les savoir-être ne seraient que peu modifiables, améliorables par le biais de la formation. Ils deviennent dès lors un requis incontournable.

- Est également justifiée par le fait que les candidats - postulant à une formation et non à un emploin'auraient pas encore acquis de compétences professionnelles entendues comme savoir et savoir-faire. Ces qualités sociales sur lesquelles se base la sélection seraient donc, en quelque sorte, les seuls éléments testables lors du recrutement.

Cependant, ces propos sont à nuancer car les candidats ont bel et bien acquis des expériences professionnelles (énoncées ci-dessus) et détiennent des diplômes (professionnels pour la plupart mais de filières très diverses) : $39 \%$ des apprentis préparant le BTS/DUT au sein de l'entreprise A ont obtenu un diplôme supérieur au baccalauréat, $11 \%$ d'entre eux, supérieur au DEUG (diplôme d'études universitaires générales). De même, $76 \%$ des candidats retenus pour préparer le CAP ont un niveau supérieur au BEPC (brevet d'études du premier cycle), $31 \%$ supérieur au baccalauréat.

Si la sélection s'opère avant tout sur les savoir-être (troisième étape de la sélection), elle s'effectue également sur les savoirs (sur le niveau de diplôme lors de la première étape, sur les savoirs de base lors de la deuxième étape) et non pas sur les savoir-faire détenus par les candidats. Ce peu de valeur accordée aux contenus des diplômes de l'enseignement professionnel (Eymard-Duvernet et Marchal, 1997) mais également aux savoirs professionnels acquis sur le marché du travail, est révélateur de la dévalorisation de la formation professionnelle et plus largement des

\footnotetext{
5 Cependant, soulignons que l'appel à des notions naturalisantes, présentées sous une forme de modernité et de scientificité dans l'optique de différencier et de hiérarchiser les individus, n’est pas un phénomène récent. Des procédés comparables par certains aspects étaient mis en œuvre au sein des écoles d'entreprise (Kergoat, 2002) ou dans le cadre de l'orientation professionnelle (Naville, 1945).
} 
savoir-faire ouvriers (Tanguy, 1998). Qu'en est-il cependant des contenus de formation délivrés aux apprentis? Quelles sont les compétences requises en fin de formation et comment sont-elles évaluées? C'est ce que nous allons observer à présent.

\section{Le « savoir-être », une composante de la connaissance}

Pour être embauché en fin de formation, les apprentis doivent, si possible, avoir obtenu leur diplôme mais surtout avoir «donné entière satisfaction à l'entreprise ». Dans une des entreprises observées, un responsable de l'apprentissage présente ainsi les compétences requises :

"On a des jeunes qui n'obtiennent pas le diplôme, alors ils recommencent, ou bien on les embauche à une catégorie inférieure. Il y a ceux qui obtiennent le diplôme mais qui ne donnent pas entière satisfaction au métier $[. .$.$] ; cela peut poser un problème mais les$ apprentis ont rarement acquis plus de $70 \%$ des compétences requises et ils sont souvent plus performants que ceux que l'on recrute directement à la sortie de l'Éducation nationale. Enfin, il y a ceux qui sont bons partout mais qui mordent tout le monde tous les matins, qui ne savent pas s'adapter, alors eux on ne peut pas les garder, c'est clair on ne les gardera pas. »

C'est ainsi que l'on embauche des apprentis qui n'ont pas obtenu leur diplôme ou qui n'ont pas acquis l'ensemble des compétences requises par l'entreprise. Derrière l'expression «donner entière satisfaction à l'entreprise », non définie clairement par les textes, ce n'est donc pas de savoir ou de savoir-faire dont il s'agit mais bien de comportement.

Afin de caractériser les contenus de formation et d'éclairer l'usage de la notion de compétence, et plus particulièrement celles de savoir-faire et de savoirêtre, nous proposons un regroupement transversal aux trois entreprises ${ }^{6}$.

Dans le cadre des formations techniques (regroupant le BEP électrotechnique de l'entreprise B et le BTS

\footnotetext{
${ }^{6} \mathrm{Vu}$ le petit nombre de formations observées (cinq), ces observations ne prétendent pas à l'exhaustivité.
}

maintenance de l'entreprise C), la technicisation des savoir-faire, l'appel aux dispositions sociales et culturelles visent à détacher les apprentis des dispositions culturelles et sociales des ouvriers pour les rapprocher de celles du technicien. Les établissements d'enseignement (lycée professionnel et CFAI - Centre de formation des apprentis de l'industrie) valorisent un apprentissage technique où le savoirfaire perd ses connotations manuelles pour désigner un mode de raisonnement centré sur la méthode qui garantirait l'adaptation des apprentis et l'efficacité de l'action.

Les formateurs en entreprise et les évaluations effectuées par les tuteurs (occupant des fonctions d'encadrement) insistent davantage que les enseignants sur la mise en pratique de ces savoirs techniques ; pourtant, ceux-ci restent éloignés des savoirs de métier mis en œuvre par les équipes de travail. C'est ainsi que les apprentis doivent abandonner leur francparler, l'argot de métier, pour adopter une tenue « correcte », un langage policé, un vocabulaire technique, tout en étant capables de présenter des exposés sur les interventions électriques devant leur hiérarchie (ceci nécessitant de rédiger un rapport, de le présenter oralement, de répondre aux questions et aux objections, d'utiliser un rétroprojecteur, etc.).

Cette recherche d'acculturation se heurte néanmoins aux pratiques des collectifs de travail qui défendent un apprentissage centré sur la figure de métier. Bien que les savoirs soient ici peu formalisés, les ouvriers développent, à tour de rôle, des pratiques collectives permettant de transmettre des connaissances, de contrôler et d'intégrer les apprentis (se prêter par exemple à des bizutages dans l'optique d'enrayer la peur du vertige : chronométrer l'apprenti lorsqu'il monte à un poteau, lui offrir un saut en parachute...). C'est que dans la culture de travail, les situations d'apprentissage relèvent d'une formation et d'une socialisation qui nécessitent l'acquisition de connaissances, de savoir-faire pratiques par imprégnation prolongée ainsi que par l'assimilation d'une discipline de travail (Hass, 1974).

\section{Naturaliser les savoirs}

Dans le cadre des formations de service (comprenant le BTS/DUT de l'entreprise A et le bac pro services de l'entreprise B) les savoirs organisationnels et les 
qualités sociales priment dans les contenus de formation et leurs évaluations. Les formateurs transmettent des règles prescrites de travail (telles que les procédures, la réglementation) associées à des savoirs sur l'entreprise (contexte juridique, connaissances des produits proposés). Ils insistent également sur nombre de qualités sociales qui impliqueraient de nouvelles dispositions (liées au développement des relations de service) non acquises par les salariés en place. Il s'agit de modeler de nouvelles postures afin de « refaire leur éducation » et d'éviter le profil type $\mathrm{du}$ «parfait titi parisien » (formateur de l'entreprise A, BTS/DUT). C'est ainsi que les formations s'appuient par exemple sur des bandes dessinées où les apprentis sont invités à repérer les postures physiques et le vocabulaire « incorrects» des personnages.

La nécessité affirmée par les entreprises, depuis une vingtaine d'années, de fonder l'acte de vente sur les relations entretenues avec les clients, conduit les enseignants de l'IUT préparant au DUT Technique de commercialisation à valoriser des qualités professionnelles telles que les capacités de démonstration, d'argumentation et de conviction (Lechaux, 1995). Ces compétences comme les items compris dans les grilles d'évaluation tels que "identifier les besoins des clients, mettre en æuvre les actions adéquates pour y répondre " (entreprise A, BTS/DUT) sont catégorisés comme des « savoirs comportementaux » et non comme des savoir-faire (acquis par le biais de la formation ou par l'expérience du travail)'.

De même, nombreux sont les enseignants préparant au bac pro services en lycée professionnel à promouvoir les qualités sociales impliquant un processus d'acculturation aux petites classes moyennes : formation aux «bonnes manières» et au «bon goût» pas cher (en conduisant par exemple les apprentis au magasin Promod, chaîne de magasin populaire pour jeunes, afin d'《 apprendre» à s'habiller). Ce diplôme, dont la spécificité est de développer des « compétences relationnelles », définies par défaut comme ne relevant pas de «savoirfaire » (Collin, 2000), renvoie à des items tels que

\footnotetext{
7 Si ce brouillage est moins présent au sein des formations techniques, nous pourrions néanmoins poursuivre le raisonnement. "Porter une tenue adaptée », dans le cadre de la formation suivie par les électriciens, est catégorisé comme un savoir-être. Ce qui là encore est fortement discutable au regard de l'importance des consignes de sécurité propres à ce métier à risque.
}

« capacités 》 à "s'intégrer au sein d'une équipe», «participer à la valorisation de l'image de l'entreprise », etc. (référentiel de diplôme, bac pro services).

Ces compétences relationnelles, bien que reconnues comme transférables à d'autres activités, n'ont cependant pas acquis le titre de techniques, ni de savoir-faire et sont associées à des qualités dites féminines, fortement naturalisées ${ }^{8}$; ces conceptions expliquent le fort taux de féminisation de ces formations ( $c f$. encadré 2) :

«On est plus exigeant pour le recrutement d'un technicien car l'on recherche des qualités intellectuelles, un potentiel pour les BEP voire des acquis théoriques et pratiques pour les bac pro techniques alors que pour le bac pro services nous nous appuyons uniquement sur la personnalité et le savoir-être du candidat [...] Je ne pense pas qu'une formation permette d'apprendre la psychologie et le contact. On peut s'améliorer mais si on n'a pas un minimum de facilités naturelles, on ne peut pas être efficace. » (cadre DRH - Direction des ressources humaines, entreprise B)

\section{Revisiter la notion de discipline}

Enfin, la formation TAD (tri-acheminement-distribution) de l'entreprise A, que nous qualifions de formation industrielle de services, recouvre essentiellement des activités de manutention (tri, acheminement) ainsi que des activités de service (distribution du courrier). Elle se caractérise par une tension entre les contenus de formation transmis en situation de travail et ceux prescrits par la direction et dispensés par les formateurs de l'entreprise.

D'un côté, des contenus de formation qui sont marqués par la reproduction d'une culture de travail. Les facteurs et les trieurs transmettent, en situation de travail, des pratiques élaborées à partir d'un ensemble d'idées partagées sur le travail. Les facteurs transmettent aux apprentis un certain nombre de règles concernant le travail bien fait, des

\footnotetext{
${ }^{8}$ C'est ce que montrent également les recherches de Josiane Pinto (1990) sur le secrétariat et de Liane Mozère (1998) sur les métiers de la petite enfance où les savoir-faire mobilisés ne sont pas considérés comme faisant partie intégrante d'un métier mais plutôt d'une condition définie par l'aptitude à être au service d'une personne.
} 
ficelles de métier propres à chaque tournée afin de faciliter le travail. Ils montrent également aux apprentis comment accroître leur autonomie (distribuer en même temps le courrier ordinaire et les recommandés) tout en traçant les limites à ne pas franchir (distribuer une lettre recommandée sans demander une pièce d'identité à un client que l'on ne connaît pas). Les situations de travail au centre de tri permettent également aux apprentis d'observer et de reproduire les pratiques effectives de freinage (partir avant l'heure sans se faire remarquer), d'aménagement des conditions de travail (trier à deux sur un même casier pour pouvoir discuter). De fait, les postiers transmettent un ensemble de pratiques professionnelles mais également une culture de travail garantissant un minimum d'autonomie et de satisfaction.

D'un autre côté, des contenus de formation qui sont empreints d'une volonté de substituer à la culture de travail une culture d'entreprise orientée (notamment) vers de nouvelles formes de disciplines. La formation TAD n'emporte cependant pas l'adhésion de l'ensemble de la Direction des ressources humaines. Pour certains, c'est "une formation de luxe», la formation traditionnelle de quelques jours étant amplement suffisante. Pour d'autres, elle revalorise deux activités centrales de l'entreprise en leur conférant le titre de métier. De plus, elle permettrait d'introduire, pour reprendre leurs expressions, « une nouvelle génération de facteurs et de trieurs » mobilisant de "nouveaux comportements professionnels bien mieux adaptés aux nouvelles missions $d u$ service public», de «faire des apprentis des agents de changement participant activement aux projets d'évolution $»$.

À l'inverse des deux cas précédents, l'ensemble de la formation professionnelle est dispensé par l'entreprise. Les contenus sont entièrement orientés vers des savoirs organisationnels (procédures, réglementation, vocabulaire technique propres à l'entreprise,

\footnotetext{
${ }^{9}$ La place accordée aux comportements des agents est un point central des réformes en matière de gestion du personnel. La compétence est définie comme un « comportement professionnel adapté »: " "L'entreprise A" poursuivra le rééquilibrage de ses critères de sélection en privilégiant les comportements professionnels et les capacités d'adaptation au regard des connaissances techniques, notamment par la définition de profils de compétences par métier» "Politique de gestion des ressources humaines », DRH, 1996, p. 21.
}

contexte juridique) et vers l'acquisition de qualités sociales (cours intégrant des jeux de rôle axés sur les relations à développer avec les clients et la hiérarchie, sur la présentation de soi...).

Les items, présents dans les fiches d'évaluation des apprentis, renvoient à un profil traditionnel d'ouvrier. Un ouvrier spécialisé à qui l'on demande de respecter les règles («retard», «absences », «probité»), de maitriser ses affects («maitrise de soi », «prend la parole de façon adaptée », " accepte la discussion ») et de mobiliser sa force de travail (« exécute une tâche jusqu'au bout», "sait rester concentré», "rapidité d'exécution »). Un ensemble de qualités qui renvoie ici à une organisation industrielle du travail axée sur la séparation entre conception et exécution, à une vision dans laquelle la hiérarchie est décisionnaire et capable de juger de la valeur, en particulier morale, des individus.

Comme dans le cadre des formations techniques, il y a coexistence, juxtaposition de deux procès d'apprentissage.

L'évaluation des qualités sociales assurerait aux entreprises l'investissement des apprentis dans leur travail et leur entreprise, l'objectif étant d'éviter les problèmes d'absentéisme, de ponctualité ou d'incivilité rencontrés par de grandes entreprises (Guy, 2002). Elle permettrait également de savoir si les apprentis intègrent les valeurs, les normes et les comportements supposés garantir leur adhésion à la place et aux missions de leur groupe professionnel définies par les profils de poste, tout en accompagnant les réformes en matière d'organisation $d u$ travail et de gestion du personnel.

Par bien des aspects, le savoir-être est très proche de la notion de discipline, des méthodes éducatives ou de management telles que l'autodiscipline, le «self governement », en œuvre dès les années 20 , dans les écoles d'entreprise, les centres d'apprentissage ou au sein des « Public Schools » anglaises.

Soulignons néanmoins que les modalités du contrôle social évoluent. À l'inverse de la discipline, le savoir-être n'apparaît pas, de prime abord, comme un moyen de coercition. Les propriétés requises ne sont plus légitimées par la morale ou par un référent idéologique quelconque mais par la science et la 
technique. D'où toute une panoplie d'outils dont l'objectif n'est plus tant d'inculquer à un groupe un comportement conforme à des règles collectives prescrites par un règlement, qu'une somme de propriétés individuelles évaluées lors d'un face-àface. Enfin, alors que les normes comportementales dans la méthode de l'autodiscipline étaient clairement distinguées de toute forme d'évaluation du travail (les savoir-faire) ou des connaissances (les savoirs), les nouveaux modes de contrôle intègrent ces différentes dimensions dans un même temps ${ }^{10}$ (Kergoat, 2001 et 2002).

Or, et c'est bien ici que se situe la distinction, la codification de la formation et de son évaluation en termes de compétences contribue non seulement à entretenir la confusion entre comportement et connaissances mais plus largement l'officialise: le savoir-être devient une composante de la connaissance à côté des savoirs et des savoir-faire. Ces différents processus conduisent à masquer nombre de savoir-faire sans cependant les exclure du champ de la formation et par-delà à revoir les qualifications à la baisse (ainsi que les formes de reconnaissance qui y sont associées), tout en faisant du temps d'apprentissage un temps de test permettant de prolonger la période d'essai.

Ces ruptures avec les normes établies sont permises non seulement par la codification de la formation et du travail en termes de compétences mais encore par la mise en œuvre de politiques de contractualisation.

\section{D'UNE POLITIQUE ÉTATIQUE À UNE POLITIQUE DE CONCERTATION}

Nous nous attachons ici à caractériser les politiques de concertation et de contractualisation menées d'une part entre les grandes entreprises et les régions

\footnotetext{
${ }^{10}$ Bien que l'institution scolaire n'évalue pas officiellement le savoir-être, nous pourrions poursuivre notre interrogation en pointant les similitudes qu'entretient cette notion avec celle de "citoyenneté à l'école » (Kergoat 2001 et 2002) ou avec 1 'introduction au collège, à la rentrée 2006 , de «la note de vie scolaire » (décret $\mathrm{n}^{\circ}$ 2006-533 paru dans le Journal Officiel du 12 mai 2006) participant à l'acquisition du BEPC (brevet des collèges).
}

et d'autre part avec le ministère de l'Éducation nationale.

Suite aux lois de 1987 et de 1992 relatives à l'apprentissage, les grandes entreprises peuvent, grâce à des conventions avec l'État ou les régions, créer des dispositifs de formation initiale nommés CFA (Centre de formation d'apprentis) « sans murs » ou encore «CFA d'entreprise». Ceux-ci, quel que soit leur statut ${ }^{11}$ et bien qu'indépendants juridiquement, sont dans les faits dépendants des entreprises. Ces dernières, et plus particulièrement les Directions des ressources humaines, fixent les directives en matière d'organisation de l'apprentissage (gestion administrative, financière et pédagogique de la formation), sélectionnent les salariés du CFA et financent son fonctionnement. Concrètement, ces CFA d'entreprise sont chargés :

- de la coordination entre les différents acteurs de l'apprentissage à un niveau local (Conseil régional, Académie, etc.) ;

- de la conclusion des conventions avec des établissements d'enseignement (lycées professionnels, IUT, universités, CFA traditionnels), aux termes desquelles ils délèguent une partie ou la totalité des enseignements tout en gardant la maîtrise administrative et pédagogique.

Dès lors, ces CFA assurent un rôle d'intermédiaire entre l'entreprise et les instances politiques et administratives.

\section{Contourner les règles nationales}

Les CFA régionaux de l'entreprise $\mathrm{C}$ permettent d'organiser des rencontres locales - au niveau de la région et du bassin d'emploi - avec le monde économique, politique, administratif, culturel ou associatif. Il s'agit, tout en recensant les besoins en apprentissage des industries agroalimentaires de la région, de créer des réseaux, de nourrir des réflexions, de constituer par les actions menées de nouvelles références en matière de relation formation-emploi, tout en organisant la mobilité de la main-d'œuvre sur le territoire.
${ }^{11}$ CFA national ou régional, lié à une entreprise, à une branche professionnelle, etc. 
Les concertations opérées entre un de ces CFA et le conseil régional de Rhône-Alpes vont permettre de constituer des Unités de formation par alternance (UFA) ; ce sont des sections d'apprentissage implantées dans des lycées professionnels mais gérées par un CFA d'entreprise ou interprofessionnel, agréé par le conseil régional. Ces UFA ont bien des atouts, en particulier le pouvoir de créer rapidement des sections d'apprentis, au moindre coût, dédiées entièrement à la formation du secteur de l'agroalimentaire dans des établissements de l'Éducation nationale disposant déjà des équipements et des enseignants. C'est ainsi qu'ils offrent la possibilité d'ajuster les flux d'apprentis et les contenus d'enseignement aux besoins de l'entreprise $\mathrm{C}$ tout en lui permettant de constituer des réseaux avec les différentes entreprises de l'agroalimentaire implantées sur le territoire.

La loi de 1982 puis la loi quinquennale de 1993 sur la régionalisation et la décentralisation accordent un important pouvoir de gestion aux régions pour mettre en œuvre une politique locale de la formation professionnelle amenant les régions à gérer les territoires nouvellement acquis en termes de rapport de force institutionnel (Moreau, 2003). Les auteurs d'un rapport sur l'apprentissage en région Rhône-Alpes (Brochier et al., 1994) soulignent les nouvelles formes de concertation et le contournement des règles édictées par l'Éducation nationale. Ils analysent également l'enjeu de ces conventions, qui déborde le simple cadre local de concertation : «Il ne $s$ 'agit pas d'expérimenter aux marges du système mais de poser les fondements d'une nouvelle régulation d'ensemble de la relation formation-emploi. » (p. 30).

C'est également au niveau du bassin d'emploi que l'entreprise $\mathrm{C}$ constitue des réseaux avec les municipalités, les entreprises locales, l'ANPE (Agence nationale pour l'emploi), des centres culturels et sportifs. Elle crée parallèlement six associations dans l'objectif affiché d'améliorer l'insertion, la formation ou l'accès à l'emploi des jeunes du bassin d'emploi. Ces rencontres locales permettent de consolider l'image de l'entreprise et d'initier des actions et réflexions en matière de relation formation-emploi (mode de financement des formations, type de contrats à valoriser, contenus de formation devant être dispensés au sein des établissements de formation, mise en œuvre des certifications d'entreprise, etc.).

Ces réseaux permettent également, sur fond de consensus social et d'actions largement médiatisées en faveur de la formation et de l'emploi, de combiner une gestion interne et une gestion externe de la maind'œuvre. De fait, l'objectif n'est pas de recruter, suite à la formation, les apprentis au sein de l'entreprise mais de les «placer» sur le territoire en fonction des besoins des clients, des sous-traitants, des fournisseurs, des PME (petites et moyennes entreprises). L'objectif est alors de disposer d'un environnement économique, industriel mais aussi social qui participe, en amont comme en aval, au développement des établissements, tout en consolidant l'image du Groupe. Les établissements contribuent dès lors au maintien sur les sites de prestataires de service, de sous-traitants comme de fournisseurs plus que jamais nécessaires dans le cadre d'une politique d'externalisation des activités « secondaires » :

«Faites un courrier aux entreprises susceptibles d'accueillir des jeunes [...] puis réunissez, à l'occasion d'un petit-déjeuner, des responsables d'entreprises pour vendre votre démarche: pensez aux fournisseurs, aux clients, aux agences d'intérim et aux sous-traitants. » (Guide de l'alternance et du tutorat, document interne, 1997)

Non seulement l'apprentissage permet d'organiser la formation et la mobilité des salariés par la constitution d'une main-d'œuvre de réserve au niveau de ses territoires et de ses établissements, mais aussi de constituer de véritables réseaux politiques, économiques et sociaux. L'apprentissage permet d'induire (que ce soit avec l'État, les régions, les syndicats, etc.) «de véritables concertations sur la qualification et la formation qui ne seraient jamais abordées autrement» (Directeur des ressources humaines, établissement de l'entreprise C).

\section{Infléchir les règles nationales}

Les CFA des entreprises A et B, contrairement à ceux du Groupe C, ont passé avec la Direction de l'Enseignement scolaire (DESCO), des conventions leur permettant d'assurer eux-mêmes une partie des formations professionnelles normalement dispensées par les établissements de l'Éducation nationale 
ou des CFA traditionnels. C'est ainsi que ces CFA d'entreprise, contrairement à ceux de l'entreprise C, organisent non seulement les séquences d'apprentissage en situation de travail mais aussi la formation dispensée en salle par des formateurs de l'entreprise. L'importance accordée aux compétences, à l'évaluation et aux contenus de formation conduit ces CFA à contrôler rigoureusement les pratiques des établissements de formation (tels que les lycées professionnels). Ils peuvent influer sur les programmes dispensés et sur les modalités d'évaluation. Pour ces entreprises, l'enjeu consiste à adapter les modes de formation et leurs contenus aux évolutions en matière d'organisation du travail et de gestion du personnel.

Il s'agit de codifier les contenus de formation et leur évaluation en termes de compétences et au-delà de rompre avec les pratiques et les représentations à l'œuvre dans les modèles antérieurs. Ces CFA d'entreprise sont dès lors conduits à créer nombre de documents (référentiel d'activité, de formation, grille d'évaluation des compétences, plan de formation interne, etc.) et à développer certaines fonctions au sein des entreprises et de leurs CFA (tuteurs, techniciens ou ingénieurs de formation). Ils ont aussi une mission d'intermédiation auprès des instances extérieures, dont l'Éducation nationale. Ce n'est sans doute pas un hasard si la direction de ces CFA est bien souvent confiée à des (anciens) salariés de l'Éducation nationale - ou détachés par celle-ciexpérimentés en matière de formation professionnelle qui occupent alors un poste d'interface déterminant lors des concertations. Ce modèle pédagogique fondé sur les compétences est caractéristique de cette recherche de mise en forme technique censée faciliter les échanges entre le monde éducatif et le monde professionnel (Ropé, Tanguy, 1994).

Les entreprises A et B, dont l'apprentissage s'inscrit dans une politique de pré-recrutement, cherchent à inscrire les concertations en matière d'offre de formation dans un cadre juridique et national. L'objectif est d'infléchir cas par cas, formation après formation, la répartition des temps, des lieux. Il s'agit de définir les contenus de formation dispensés en son sein, d'influer sur ceux délivrés par l'Éducation nationale, voire d'intégrer des évaluations effectuées en entreprise dans le processus de délivrance des diplômes ( $c f$. encadré 3).

Le bac pro services et le CAP TAD renvoient à des profils de diplôme très différents. Le premier est un diplôme volontairement large, censé répondre aux attentes d'entreprises différentes. À l'inverse, le CAP $\mathrm{TAD}$, à la seule initiative de l'entreprise $\mathrm{A}$, est un diplôme spécifique, pensé par et pour cette dernière, relativement proche des certifications de branches. Cependant, et malgré leurs différences, nous assistons dans l'un et l'autre cas à un désengagement de l'Éducation nationale, non pas envers la transmission des savoir-faire (nombreux sont ceux à ne pouvoir être transmis qu'en situation de travail) mais dans leur définition, évaluation et validation (Stroobant, 1993). C'est par exemple le cas des savoir-être ou des compétences comportementales qui ne sont ni définis, ni objectivés et dont l'évaluation repose plus souvent sur un jugement que sur une mesure $(C f$. Dejours, 2003).

\section{UNE CONTRACTUALISATION DE L'APPRENTISSAGE}

La démarche comparative permet dès lors de montrer que ces entreprises mettent en place des stratégies différentes pour tendre vers un même objectif. Alors que l'entreprise $\mathrm{C}$ tente de contourner les normes établies par l'Éducation nationale en créant un marché régional de l'apprentissage, les entreprises $\mathrm{A}$ et $\mathrm{B}$ cherchent à inscrire durablement les modifications opérées, à leur initiative, dans un cadre juridique et national. Ces politiques ont pour point commun d'induire des concertations avec l'État en matière de formation professionnelle tout en étant initiées non par des branches professionnelles mais par des entreprises singulières.

Les relations entretenues entre les grandes entreprises d'une part et les instances régionales ou étatiques d'autre part, relèvent d'une même logique, celle d'une contractualisation de l'apprentissage. Si les lois relatives à l'apprentissage offrent un cadre général, la modulation des temps, lieux et contenus de formation se négocie de plus en plus dans un cadre 


\section{Encadré 3 \\ Redéfinir l'offre de formation : concertation autour de deux diplômes}

Le CAP TAD (Tri-Acheminement-Distribution) a été créé par et pour la seule entreprise A. Le corps de l'Éducation nationale ne comportant pas d'enseignants formés à ces activités, la CPC* compétente a choisi de confier aux formateurs de l'entreprise le soin de dispenser et d'évaluer l'ensemble des enseignements techniques et professionnels. De plus, l'entreprise recrutant essentiellement des candidats ayant obtenu un premier CAP, la validation des enseignements généraux n'a pas lieu d'être. Les apprentis préparent donc leur diplôme en un an et ne passent que 224 heures en lycée professionnel (le minimum, selon la loi, étant de 400 heures annuelles) et 1453 heures en entreprise.

Enfin, l'entreprise A obtient la mise en place du contrôle continu qui permet d'intégrer les évaluations effectuées par les tuteurs en entreprise. Ainsi, sur les trois épreuves professionnelles notées sur 300 points, les évaluations effectuées par les tuteurs sont comptabilisées sur 180 points et les évaluations des formateurs de l'entreprise sur 120 points.

Le calcul est rapide : I'entreprise délivre la totalité des points nécessaires pour obtenir le CAP TAD. Dès lors, ceci constitue une rupture avec l'esprit de la loi du 4 octobre 1943 qui confie à l'État le monopole de la délivrance des diplômes.

Le bac pro services, à l'instigation de nombreuses entreprises de secteurs différents posait, quant à lui, deux types de problèmes à la CPC concernée : d'une part, l'explicitation des attentes des entreprises en matière de comportements et la formalisation du diplôme en techniques relationnelles; d'autre part, l'absence d'enseignants formés à ces techniques. La construction de ce diplôme marque, comme dans le cadre du CAP TAD, un retrait de l'Éducation nationale quant à l'accompagnement de la formation. Les CPC n'ont pas formalisé la demande des entreprises en termes de compétences relationnelles (les items restent très proches des compétences comportementales telles qu'elles sont définies par l'entreprise B pour cette même formation, cf. première partie), situation qui conduit de facto à déléguer cette partie de la formation aux entreprises: "L'adaptation du jeune à la nature spécifique du service (techniques relationnelles) aurait à se faire dans l'entreprise et pourrait être acquise par le biais de périodes en entreprise, par l'apprentissage, en contrat de qualification. » (Inspectrice générale de l'Éducation nationale) in Le Tiec (1995).

$\left(^{*}\right)$ : Les commissions professionnelles consultatives (CPC) regroupent l'ensemble des partenaires sociaux chargés de la création et de l'élaboration des diplômes professionnels et techniques (représentants des employeurs, des salariés, des pouvoirs publics et personnes qualifiées).

d'engagements réciproques, par le biais de contrats ou de conventions qui permettent à des entreprises singulières d'exprimer leurs intérêts particuliers.

Si les régions affirment leur volonté quant à l'organisation de l'apprentissage sur leur territoire, l'État occupe lui une position ambivalente : d'un côté, il affirme sa volonté d'aligner l'apprentissage sur le modèle scolaire ; de l'autre, il offre un cadre suffisamment souple pour permettre une contractualisation. Ces dispositifs deviennent un outil parmi d'autres d'une stratégie de contournement du monopole public (et dans certains cas de la négociation entre partenaires sociaux) grâce aux relations entretenues entre sphère éducative et sphère économique.

Dans les deux cas, cette pluralité de normes éminemment variables contribue à faire de l'apprentissage un lieu d'expérimentation qui échappe aux procédures régulières de définition de l'offre de formation tout en posant les fondements d'une nouvelle relation en matière de formation, d'emploi et de qualification. 
Bien que les politiques d'apprentissage soient marquées par une grande variabilité (rendue en partie possible par les politiques de contractualisation), les processus décrits permettent d'éclairer quelques-uns des changements en matière de politiques de formation. Ces changements, initiés par l'Éducation nationale et par de grandes entreprises dont la politique est dirigée par les directions nationales, permettent des expériences en rupture avec les normes établies de longue date.

La codification du travail et de la formation en termes de compétences conduit à ce que le savoirêtre devienne une composante de la connaissance à côté des savoirs et des savoir-faire. La primauté accordée aux qualités sociales permet de départager une population de candidats déjà diplômée, de natu- raliser des savoir-faire tout en réhabilitant la notion de discipline. Cette construction d'outils destinés à mesurer les qualités sociales des salariés et la mise en œuvre de politiques de contractualisation participent à un contournement des négociations propres aux branches professionnelles, à une remise en cause des formes codifiées dans les conventions collectives à partir des diplômes. Certes, l'École reste le lieu où se définissent les politiques éducatives. Cependant, les trois grandes entreprises étudiées tendent à remettre en cause le rôle déterminant conféré par l'Éducation nationale aux diplômes dans l'organisation de la formation professionnelle, et plus largement dans la production des hiérarchies sociales et professionnelles.

\section{BIBLIOGRAPHIE}

Bourdieu P. (1979), La distinction, Paris, Éditions de Minuit, $670 \mathrm{p}$.

Brochier D., Causse L., Richard A., Verdier E. (1994), « L'apprentissage coopératif en Rhône-Alpes (1988-1993) », rapport Céreq, Étude n 66, 81 p.

Brucy G. (1998) « Histoire des diplômes de l'Enseignement technique et professionnel (1880-1965). L'État, l'École, les Entreprises et la certification des compétences », Paris, Belin.

Collin C. (2000), « Évaluation du BAC professionnel Services », CPC Documents.

Dejours C. (2003), «L'évaluation au travail à l'épreuve du réel », INRA Éditions, 82 p.

Dubernet A.-C. (1996), «La sélection des qualités dans l'embauche », Formation Emploi, n ${ }^{\circ}$ 54, pp. 314.
Eymard-Duvernet F., Marchal E. (1997), Façon de recruter, les jugements des compétences sur le marché du travail, Paris, Metaillé, 239 p.

Guy T. (2002), "L'intégration, une phase de l'insertion », Formation Emploi, n ${ }^{\circ} 77$, pp. 21-36.

Hass J. (1974), "The stages of the highsteel iron worker, apprentice career", The sociological quarterly, $\mathrm{n}^{\circ} 15$, winter, pp. 486-493.

Hatzfeld N. (1989), "L'école d'apprentissage Peugeot (1930-1970): une formation d'excellence», Formation Emploi, $\mathrm{n}^{\circ}$ 27-28, pp. 115-130.

Kergoat P. (2001), «Savoirs, qualifications, compétences : enjeux pour l'entreprise, enjeux pour l'école» in "Apprendre autrement aujourd'hui ?» Cité des sciences et de l'industrie, http://education.cite-sciences.fr/education/apprendre/savoirs p1htm. 
Kergoat P. (2002), L'apprentissage dans les grandes entreprises (En France). Étude de trois cas, Thèse de doctorat de sociologie sous la direction de L. Tanguy, Paris X-Nanterre.

Kergoat P. (2006), « De l'indocilité au travail d'une fraction des jeunesses populaires. Les apprentis et la culture ouvrière », Sociologie du travail, $\mathrm{n}^{\circ} 48$, pp. 545-560.

Lechaux P. (1995), « Alternance et jeux des acteurs. L'exemple du baccalauréat professionnel », Formation Emploi, n' 49, pp. 47-68.

Lamanthe A., Verdier E. (1999), «La décentralisation de la formation professionnelle des jeunes: la cohérence problématique de l'action publique » (avec A. Lamanthe), Sociologie du Travail, $\mathrm{n}^{\circ} 4$, pp. 1-25.

Le Tiec C. (1995), « De l'évaluation à la prospective. Compte rendu d'une table ronde», Formation Emploi, $\mathrm{n}^{\circ}$ 52, pp. 101-111.

Linhart D. (1994), « Le taylorisme n'est pas mort», Sciences humaines, $\mathrm{n}^{\circ} 38$.

Moreau G. (2003), Le Monde Apprenti, La Dispute, Paris, $272 \mathrm{p}$.
Mozére L. (1998), «Les métiers de la crèche. Entre compétences féminines et savoirs spécialisés", Cahiers du GEDISST, n ${ }^{\circ} 22$, pp. 105-123.

Naville P. (1945), Théorie de l'orientation professionnelle, Paris, Gallimard, 290 p.

Pinto J. (1990), «Une relation enchantée : la secrétaire et son patron», Actes de la Recherche en Sciences Sociales, $\mathrm{n}^{\circ} 84$.

Quenson E. (2001), Les écoles d'apprentissage Renault, CNRS Éditions, $307 \mathrm{p}$.

Ropé F., Tanguy L. (1994), Savoirs et compétences, Paris, l'Harmattan, 243 p.

Stroobants M. (1993), Savoir-faire et compétences au travail, Bruxelles, université de Bruxelles, 383 p.

Tanguy L. (1998), « Du système éducatif à l'emploi. La formation un bien universel ? ", Cahiers français, $\mathrm{n}^{\circ} 285$, pp. 98-107.

Tanguy L. (2002), « La mise en équivalence de la formation avec l'emploi dans les IVe et Ve Plans (1962-1970) », Revue française de sociologie, $\mathrm{n}^{\circ} 43$ 44, pp. 685-709.

Tanguy L. (2005), « De l'éducation à la formation : quelles réformes?», Éducation et Sociétés, n 16, pp. 99-102. 


\section{Résumé}

\section{Une redéfinition des politiques de formation. Le cas de l'apprentissage dans les grandes entreprises}

Prisca Kergoat

Nous nous proposons d'examiner quelques dimensions des changements à l'œuvre en matière de politiques de formation professionnelle. Nous montrerons que les qualités sociales, prépondérantes dans le cadre des formations par apprentissage, deviennent une composante de la connaissance à côté des savoirs et des savoir-faire. Cette rupture est permise non seulement par la codification de la formation et du travail en termes de compétences mais encore par les politiques de contractualisation menées entre de grandes entreprises et l'Éducation nationale ou les régions. Ces changements contribuent à faire de l'apprentissage un lieu d'expérimentation en posant les fondements d'une nouvelle relation en matière de formation, d'emploi et de qualification.

\section{Mots-clés}

Apprentissage, contenu de formation, formation professionnelle en entreprise, politique de formation professionnelle

Journal of Economic Literature : M 53, M 55, I 28 